\title{
L'interféron $\alpha$, le plus court chemin entre la membrane et les gènes
}

La transduction d'un signal perçu à la membrane cellulaire vers l'appareil transcriptionnel peut emprunter différentes voies. L'une des mieux connues est celle des récepteurs couplés aux protéines $G$, dont l'activation entraîne la libération d'un second messager, par exemple l'AMP cyclique, l'inositol triphosphate, le di-acyl-glycérol, le calcium, etc. Ces messagers activent euxmême un système biologique, le plus souvent une protéine kinase, ce qui peut alors entraîner un changement de l'état de phosphorylation de facteurs de transcription dont l'activité et ou la localisation subcellulaire seront modifiées. Rien de tel n'avait cependant été jusqu'alors décrit concernant les signaux empruntant la voie des tyrosine kinases. Des progrès récents ont indiqué que, dans de nombreux cas, ces signaux semblaient emprunter une voie passant par les protéines de la famille p21 ras [1]. Dans d'autres cas, des résultats suggéraient qu'une phosphorylation sur des tyrosines de facteurs de transcription pouvait également être en cause ; c'est ainsi que la stimulation de cellules par le facteur IGF-1 (insulin growth factor 1) entraîne l'apparition de protéines nucléaires phosphorylées sur des tyrosines, l'une de ces protéines étant le produit de l'oncogène c-jun $\left(\mathrm{m} / \mathrm{s} n^{\circ} 3\right.$, vol. 8 , p. 280). C'est maintenant la démonstration que des facteurs de transcription peuvent être directement activés par l'intermédiaire d'une tyrosine kinase, qui vient d'être faite par plusieurs équipes travaillant sur l'induction transcriptionnelle sous l'action des interférons (IFN). Les gènes répondant aux interférons de type 1 ( $\alpha$ et $\beta$ ) possèdent dans leur région régulatrice un élément dénommé ISRE (interferon sensitive response element) capable de fixer, dans les cellules traitées par l'IFN $\alpha / \beta$, un complexe dénommé ISGF 3. ISGF 3 est composé de quatre types de sous-unités : l'une de $48 \mathrm{kDa}$, dotée d'une activité propre de fixation à augmente sous l'action d'un traitement par l'IFN $\gamma$, ce qui justifie sa désignation d'ISGF $3 \gamma$. Les trois autres sousunités sont collectivement dénommées ISGF $3 \alpha$; elles sont de 113, 91 et $84 \mathrm{kDa}$. Lors de la stimulation par IFN $\alpha$, les trois sous-unités ISGF $3 \alpha$ sont transloquées du cytoplasme vers le noyau et, associées à ISGF $3 \gamma$, elles se fixent à ISRE. En présence d'ISGF $3 \alpha$, l'affinité d'ISGF $3 \gamma$ pour ISRE est multipliée près de 20 fois. Cette réponse transcriptionnelle à l'interféron est extrêmement rapide, ce qui suggérait qu'elle empruntait un chemin relativement direct.

Plusieurs chercheurs du laboratoire de J. E. Darnell (New York, USA) viennent de cloner les ADNc codant pour les quatre sous-unités d'ISGF [2-4]. Les sous-unités ISGF $3 \alpha$ de 91 et $84 \mathrm{kDa}$ sont codées par un même gène et ne diffèrent que par leurs extrémités carboxyterminales. En revanche, la sous-unité de $113 \mathrm{kDa}$ est codée par un autre gène de la même famille, dont la séquence traduite est à $40 \%$ analogue à celle des deux autres sousunités. Ces trois protéines comportent de nombreuses caractéristiques des facteurs de transcription : présence de fermeture à glissière de leucine (leucine zipper), de motifs HLH (helix loop helix) et de domaines acides évoquant des régions d'activation transcriptionnelle. De plus, X. Y. Fu, initialement dans le laboratoire de J. E. Darnell mais ayant maintenant créé sa propre équipe, également à New York, a démontré que les trois sous-unités ISGF $3 \alpha$ possèdent des domaines $\mathrm{SH} 2$ et $\mathrm{SH} 3$ (src homologies 2 et 3). Les domaines $\mathrm{SH} 2$ semblent caractériser les protéines capables d'interagir avec des résidus phosphotyrosines et des tyrosine kinases, alors que les domaines $\mathrm{SH} 3$, souvent associés aux précédents, pourraient permettre des relations particulières avec des protéines du cytosquelette [1]. Ces sous-unités ISGF $3 \alpha$ possèdent également des résidus tyrosines extrêmement bien conservés. D'ail- leurs, elles sont phosphorylées sur ces tyrosines après traitement des cellules par l'IFN $\alpha$, et cette phosphorylation est inhibée par des inhibiteurs de protéine kinases (staurosporine et génistéine) $[5,6]$. Il est possible de reproduire, dans un système acellulaire in vitro, la phosphorylation des sous-unités ISGF $\alpha$ en présence d'IFN $\alpha[5,7]$. Cette phosphorylation semble se dérouler au niveau de la membrane cellulaire [7] et entraîne la formation d'un complexe composé de ces trois sousunités. Le traitement par une protéine phosphatase, susceptible de déphosphoryler les résidus phosphotyrosines, empêche la formation du complexe ISGF $3 \alpha$. Des phénomènes de même nature pourraient expliquer l'action de l'interféron $\gamma$ : des cellules stimulées par cet interféron phosphorylent la sous-unité de $91 \mathrm{kDa}$ d'ISFG $\alpha$, mais non les deux autres sous-unités. Les deux voies de signalisation interféronique $\alpha$ et $\gamma$ pourraient donc mettre en commun certaines de leurs molécules, probablement associées à des complexes transcriptionnels différents, aboutissant à la stimulation d'éléments de réponse également différents [6]. Il restait à comprendre le mécanisme de l'induction par l'interféron de la phosphorylation de résidus tyrosines des sous-unités ISGF $3 \alpha$. Cela n'était pas évident puisque, contrairement à des récepteurs comme ceux de l'insuline, d'IGF 1, d'EGF, de PDGF ou de FGF, les récepteurs des interférons ne sont pas dotés, par eux-même, d'activité tyrosine kinase. Le problème a été résolu au terme d'un long travail, extrêmement impressionnant, de Sandra Pellegrini et de ses collègues travaillant initialement dans le laboratoire de G. R. Stark (ICRF, Londres, GB) puis dans l'unité Inserm dirigée par Marc Fellous à l'Institut Pasteur de Paris. En Angleterre, S. Pellegrini avait isolé, grâce à d'ingénieuses expériences de génétique somatique, des cellules mutées ne répondant plus aux interférons $\alpha$ et $\beta$, mais possédant un 
récepteur apparemment normal, quoique son affinité pour IFN $\alpha$ fût diminuée [8]. La transfection de ces cellules par un vecteur d'expression commandant la synthèse du récepteur ne rétablissait d'ailleurs pas la réponse à l'IFN $\alpha$. La méthode utilisée alors avait consisté à introduire dans des cellules déficientes en HGPRT (hypoxanthine guanine phosphoribosyl transférase), un gène gpt (guanine phosphoribosyl transférase) placé sous le contrôle d'éléments ISRE. Ce gène code pour une enzyme d' $E$. coli permettant de compenser le déficit en HGPRT ; lorsqu'il est actif, les cellules HGPRT $^{-}$peuvent pousser sur milieu HAT (hypoxanthine-aminoptérinethymidine), mais sont détruites en présence de 6-thioguanine (6 TG). Par conséquent, les cellules mutantes isolées par S. Pellegrini ne poussent pas sur milieu HAT, même en présence d'interféron, mais sont résistantes au 6 TG, même en présence d'interféron. Dans un très récent travail, signé par L. Velazquez, de l'équipe de S. Pellegrini dans le laboratoire de M. Fellous, en collaboration avec G. R. Stark, est rapporté le clonage d'un gène permettant de restituer à cette lignée mutée la sensibilité à l'interféron $\alpha$, et donc la résistance en milieu HAT plus IFN $\alpha$ [9]. En bref, ces cellules ont été transfectées par de l'ADN humain et par un vecteur d'expression conférant la résistance au G 418 dans les cellules eucaryotes et à la kanamycine dans les cellules procaryotes (gène neo). Une sélection par le G 418 permit alors d'isoler une vingtaine de milliers de clones supposés contenir des fragments d'ADN humain associés de manière covalente au gène neo, qui sert ainsi d'étiquette. Dans une seconde étape, les cellules furent placées sur milieu HAT et traitées par l'interféron : seules celles ayant récupéré une réponse à IFN $\alpha$ sont censées survivre dans ces conditions. L'ADN de ces cellules révertantes répondant à l'interféron fut alors isolé et servit à transfecter d'autres cellules mutantes à partir desquelles d'autres révertants furent à nouveau sélectionnés. Une banque de cosmides fut ensuite fabriquée avec l'ADN de ces cellules, dont on sélectionna les clones résistant à la kanamycine, par conséquent possédant un gène neo et, de ce fait, l'ADN humain $m / s n^{\circ} 8$, vol. 8, actobre 92

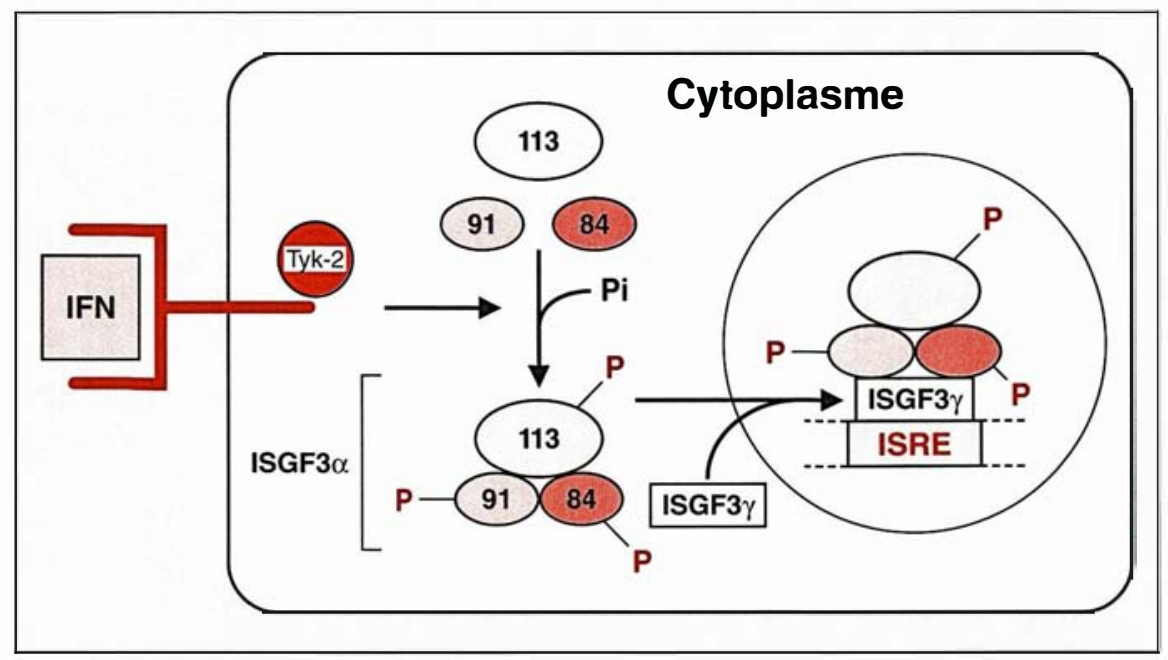

Figure 1. Schéma de la réponse transcriptionnelle aux interférons $\alpha$ et $\beta$. La fixation d'IFN $\alpha$ à son récepteur entraîne, par l'intermédiaire de la tyrosine kinase Tyk-2, la phosphorylation de résidus tyrosines au niveau des trois sous-unités ISGF $3 \alpha$. Celles-ci forment alors un complexe qui se fixe à I'ISGF $\gamma$ de $48 k D a$, le tout étant transloqué dans le noyau. L'affinité de ce complexe pour l'ISRE est au moins 20 fois supérieure à celle de I'ISGF $\gamma$ seul. De plus, les sous-unités ISGF $3 \alpha$ possèdent des domaines d'activation transcriptionnelle qui permettent la stimulation de la transcription de gènes avoisinants.

complémentant la mutation. L'ADN d'un cosmide ainsi obtenu, dont on vérifia qu'il était lui-même capable de complémenter les cellules mutantes, servit à isoler des ADN complémentaires codant pour une tyrosine kinase, produit du gène $t y k-2$, qui avait été précédemment décrite mais dont l'activité physiologique restait inconnue [10]. Cette remarquable série de travaux permet par conséquent de préciser maintenant le schéma de l'activation transcriptionnelle secondaire à l'action des interférons $\alpha$ et $\beta$, et probablement de l'interféron $\gamma$, ainsi que cela est schématisé sur la figure 1. L'interféron se fixe à son récepteur membranaire qui est ainsi activé et qui interagit, directement ou indirectement, avec la tyrosine kinase Tyk-2. Celle-ci subirait alors une autophosphorylation sur une tyrosine, permettant une interaction étroite avec les domaines $\mathrm{SH} 2$ des sous-unités ISGF $3 \alpha$, probablement localisées à la face interne de la membrane cellulaire. La phosphorylation sur un résidu tyrosine de ces sousunités entraîne la formation d'un complexe qui s'associe à la protéine ISGF $\gamma$ de $48 \mathrm{kDa}$, l'ensemble étant transloqué dans le noyau et se fixant à l'élément d'ADN ISRE. La forma- tion de ce complexe permet alors l'activation transcriptionnelle. L'interféron $\gamma$ se fixant à son récepteur agirait de la même manière, mais le produit de tyk-2 n'est probablement pas ici impliqué car la sensibilité à l'IFN $\gamma$ des cellules mutantes de S. Pellegrini n'était pas modifiée ([8] et communication personnelle). IFN $\gamma$ entraîne la phosphorylation de la sous-unité de $91 \mathrm{kDa}$ d'ISGF $3 \alpha$, qui participe probablement à un complexe particulier permettant la fixation sur un élément de réponse à l'interféron $\gamma$ différent de l'ISRE, et par conséquent l'activation transcriptionnelle de gènes spécifiques d'IFN $\gamma$. L'un de ces gènes pourrait d'ailleurs être celui codant pour ISGF $\gamma$. Cet ensemble de résultats, d'une part éclaire d'un jour tout nouveau les mécanismes d'action des interférons, ces cytokines décidément jamais " démodées ", qu'il s'agisse de leurs rôles physiologiques, de leurs potentialités thérapeutiques ou de l'exemplarité de leurs modes d'action; d'autre part, il attire l'attention sur la possibilité d'une transmission très directe à la machinerie transcriptionnelle, de signaux perçus par des récepteurs membranaires, la tyrosine kinase Tyk-2 étant ici, peut-être, le seul intermé- 
diaire. Des expériences complémentaires nous indiquerons probablement bientôt si la route peut être encore plus courte, par contact direct entre un récepteur doté de sa propre activité de tyrosine kinase et des facteurs de transcription, comme cela est d'ailleurs suspecté depuis longtemps. Ces récepteurs pourraient ainsi informer les systèmes de régulation génique: directement, par phosphorylation de composants de l'appareil de transcription ; et indirectement, par l'intermédiaire du système des petites protéines $G$ p21 ${ }^{\text {ras }}[1]$, participant ainsi au contrôle de phases multiples de l'activité cellulaire

Axel Kahn

\section{RÉFÉRENCES}

1. Kahn A. Les protéines Ras et GAP, des relais sur la voie de transmission du signal passant par l'activation des tyrosine kinases. médecine/sciences $1992 ; 8: 471-5$.

2. Fu XY, Schindler C, Improta T, Aebersold RH, Darnell JE. The proteins of ISGF 3, the IFN $\alpha$ induced activator, define a new gene family of signal transducers. Proc Nat Acad Sci USA 1992 (sous presse).

3. Schindler C, Fu XY, Improta $\mathrm{T}$, Aebersold RH, Darnell JE. The proteins of ISGF 3 : one gene encodes 91 and $84 \mathrm{kDa}$ ISGF $3 \alpha$ proteins. Proc Natl Acad Sci USA 1992a (sous presse).

4. Veals SA, Schindler C, Leonard D, et al. Subunit of an IFN $\alpha$ responsive transcription factor is related to IRF and Myb families of DNA-binding proteins. Mol Cell Biol 1992 (sous presse).

5. Fu XY. A transcription factor with $\mathrm{SH} 2$ and SH3 domains is directly activated by an interferon $\alpha$-induced cytoplasmic protein tyrosine kinase(s). Cell 1992; $70: 323-35$

6. Schindler C, Shuai K, Prezioso VR, Dar nell JE. Interferon-dependent tyrosine kinase activates latent cytoplasmic transcription factors. Science 1992 ; 257 : 809-13.

7. David M, Larner AC. Activation of transcription factors by interferon $\alpha$ in a cell-free system. Science $1992: 257: 813-5$

8. Pellegrini S, John J, Shearer M, Kerr IM, Stark GR. Use of a selectable marker regulated by $\alpha$ interferon to obtain mutations in the signaling pathway. Mol Cell Biol 1989 ; 9 : 4605-12

9. Velazquez L, Fellous M, Stark GR, Pellegrini S. A protein tyrosine kinase in the interferon $\alpha / \beta$ signaling pathway. Cell 1992 ; $70: 313-22$.

10. Krolewski JJ, Lee R, Eddy R, Shows TB, Dalla-Favera R. Identification and chromosomal mapping of new human tyrosine kinase genes. Oncogenes $1990 ; 5$ : 277-82.

TIRÉS A PART

A. Kahn.

\section{LE PRIX NOBEL DE MÉDECINE 1992}

\author{
Phosphorylation réversible des protéines
}

Le Prix Nobel 1992 de médecine ou de physiologie a été décerné à Edmond $\mathrm{H}$. Fischer, né le 6 Avril 1920 à Shanghai (Chine), et à Edwin Krebs, né le 6 Juin 1918 à Lansing, lowa, USA.

Tous deux travaillent depuis longtemps à I'Université Washington de Seattle (WA, USA). Le premier article marquant à la fois leur coopération et le début des travaux qui allaient leur valoir le Prix Nobel a été publié dans Journal of Biological Chemistry en 1955 [1]. Ce travail s'inscrit dans une ligne de recherche dont les grands noms sont les Cori, Carl et Gerti, et l'un de leurs élèves, comme Edwin Krebs lui-même, Earl W. Sutherland. Les Cori avaient découvert l'interconversion de la phosphorylase musculaire entre une forme $b$, inactive, et $a$, active, ce qui allait leur valoir le prix Nobel en 1947. Sutherland, quant à lui, reçu le Prix Nobel en 1971 pour la découverte de l'AMP cyclique. Fisher et Krebs démontraient, dans leur article de 1955 , que l'interconversion de la phosphorylase était due à une réaction de phosphorylation aboutissant à la forme active et de déphosphorylation la convertissant en la forme b inactive. La même année, Sutherland parvenait aux mêmes résultats avec la phosphorylase hépatique [2]. En 1959, Fischer et Krebs démontraient que l'enzyme responsable de la phosphorylation de la phosphorylase, la phosphorylase kinase, était elle-même activée par phosphorylation [3]. Puis ce fût, en 1968, la découverte par Krebs que c'était I'AMP cyclique qui activait la phosphorylase kinase [4], tandis que Fisher consacrait une grande partie de ses recherches à l'étude de la protéine kinase activée par l'AMP cyclique. Alors qu'il avait fallu 14 ans, de 1955 à 1969 [5], pour observer la régulation par phosphorylation d'autres voies métaboliques que celle de la synthèse et de la dégradation du glycogène, les années qui suivirent montrèrent que la phosphorylation réversible des protéines était la voie la plus universelle de régulation de leur activité, c'est-à-dire de réponse aux signaux multiples intra- et extracellulaires. C'est dire combien sont mérités ces Prix Nobel dont Jacques Demaille parlera plus longuement dans notre prochain numéro. Au tout début des recherches des Cori sur la phosphorylase, qui aurait prédit que ces travaux allaient conduire, directement ou indirectement, à cinq prix Nobel ? Voilà de quoi remplir d'espoir tous ceux à qui l'on reproche parfois le caractère ésotérique de leurs recherches.

Axel Kahn

[1. Fischer EH, Krebs EG. J Biol Chem 1955; 216 : 121-32.]

[2. Sutherland EW, Wosilait WD. Nature 1955; $175: 169-71$.

[3. Krebs EG, et al. J Biol Chem 1959 ; 234 : 2867-73.]

14. Walsh DA, et al. J Biol Chem 1968; $243: 3763-5$.

[5. Linn TC, et al. Proc Natl Acad Sci USA $1969 ; 62$ : 234-41.] 